THÉORIE DES NOMBRES

BESANÇON

La norme des espaces quadratiques et la forme trace des algèbres simples centrales 


\title{
La norme des espaces quadratiques et la forme trace des algèbres simples centrales
}

\author{
Jean-Pierre Tignol* \\ IRMAR - Université de Rennes I \\ Campus de Beaulieu \\ 35042 Rennes Cedex
}

Le transfert (de Scharlau) associe à tout espace quadratique sur un corps $K$, extension finie d'un corps $k$, un espace quadratique sur $k$ moyennant le choix d'une forme linéaire $s: K \rightarrow k$. Cette construction bien connue (voir [2, Chap. 7], [8, Chap. 2, §5]) rend d'importants services en théorie algébrique des formes quadratiques.

L'objet de cet article est d'attirer l'attention du lecteur sur une version multiplicative du transfert de Scharlau. Plusieurs propriétés importantes du transfert, telles que l'additivité ou la compatibilité avec l'équivalence de Witt, font défaut à cette version multiplicative; cependant la dernière partie de ce travail montre que celle-ci apparaît de manière naturelle en relation avec certaines formes hermitiennes dans le calcul de l'algèbre de Clifford de la forme $\operatorname{Trd}_{A}\left(x^{2}\right)$, où $\operatorname{Trd}_{A}$ est la trace réduite d'une algèbre simple centrale A.

Pour simplifier, et parce que ce cas est suffisant pour l'application envisagée, seul le cas où l'extension $K / k$ est quadratique est traité ici.

Dans tout ce travail, la caractéristique du corps de base est supposée différente de 2. Si $\varphi$ est une forme quadratique sur un corps $k$, on note dét $\varphi$ l'élément de $k^{\times} / k^{\times 2}$ représenté par le déterminant de la matrice de $\varphi$ par rapport à une base quelconque. Le discriminant de $\varphi$ est défini par:

$$
\operatorname{disc} \varphi=(-1)^{n(n-1) / 2} \operatorname{dét} \varphi \in k^{\times} / k^{\times 2} .
$$

\section{La norme et la trace des espaces quadratiques}

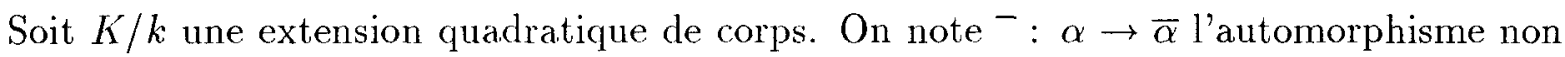
trivial de $K / k$.

$\grave{A}$ tout espace vectoriel $V$ sur $K$, on associe un nouvel espace vectoriel

$$
\bar{V}=\{\bar{v} \mid v \in V\},
$$

où l'addition et la multiplication scalaire sont définies par:

$$
\bar{v}+\bar{w}=\overline{v+w} \quad \bar{v} \alpha=\overline{v \bar{\alpha}} \quad \text { pour } v, w \in V \text { et } \alpha \in K .
$$

* Subventionné en partie par le Fonds National de la Recherche Scientifique (Belgique). 
On définit alors la norme de $V$ : c'est l'espace vectoriel sur $k$ des éléments de $\bar{V} \otimes_{K} V$ invariants sous l'homomorphisme d'échange

$$
\dot{e}: \bar{V} \otimes_{K} V \rightarrow \bar{V} \otimes_{K} V
$$

défini par:

$$
\dot{e}(\bar{v} \otimes w)=\bar{w} \otimes v \quad \text { pour } v, w \in V
$$

Donc,

$$
N_{K / k}(V)=\left\{x \in \bar{V} \otimes_{K} V \mid \dot{e}(x)=x\right\} .
$$

La multiplication scalaire dans $\bar{V} \otimes_{K} V$ définit un isomorphisme canonique d'espaces vectoriels sur $K$ :

$$
N_{K / k}(V) \otimes_{k} K \stackrel{\sim}{\rightarrow} \bar{V} \otimes_{K} V
$$

En particulier,

$$
\operatorname{dim}_{k} N_{K / k}(V)=\left(\operatorname{dim}_{K} V\right)^{2} .
$$

Si $b: V \times V \rightarrow K$ est une forme bilinéaire, on définit une forme bilinéaire

$$
\bar{b}: \bar{V} \times \bar{V} \rightarrow K
$$

par:

$$
\bar{b}(\bar{v}, \bar{w})=\overline{b(v, w)} \quad \text { pour } v, w \in V .
$$

Soit à présent $\varphi: V \rightarrow K$ une forme quadratique et soit $b$ sa forme bilinéaire associée:

$$
b(v, w)=\frac{1}{2}[\varphi(v+w)-\varphi(v)-\varphi(w)] \quad \text { pour } v, w \in V .
$$

La norme de $\varphi$, notée $N_{K / k}(\varphi)$, est alors définie comme la restriction à $N_{K / k}(V)$ de la forme quadratique $\bar{\varphi} \otimes \varphi$ sur $\bar{V} \otimes_{K} V$ associée à la forme bilinéaire $\bar{b} \otimes b$. Par exemple, pour $v, w \in V$,

$$
N_{K / k}(\varphi)(\bar{v} \otimes w+\bar{w} \otimes v)=\overline{\varphi(v)} \varphi(w)+\overline{\varphi(w)} \varphi(v)+2 N_{K / k}(b(v, w)) .
$$

Les propriétés suivantes sont des conséquences directes de la définition, dont la démonstration est laissée au lecteur:

1.1 Propriétés. a) L'isomorphisme canonique $N_{K / k}(V) \otimes_{k} K=\bar{V} \otimes_{K} V$ induit une isométrie:

$$
\left(N_{K / k}(V), N_{K / k}(\varphi)\right) \otimes_{k} K=\left(\bar{V} \otimes_{K} V, \bar{\varphi} \otimes \varphi\right) .
$$

b) Si $(V, \varphi)$ et $(W, \psi)$ sont des espaces quadratiques sur $K$, il y a une isométrie canonique:

$$
\left(N_{K / k}\left(V \otimes_{K} W\right), N_{K / k}(\varphi \otimes \psi)\right)=\left(N_{K / k}(V), N_{K / k}(\varphi)\right) \otimes_{k}\left(N_{K / k}(W), N_{K / k}(\psi)\right) .
$$

c) L'isomorphisme canonique $\bar{V} \otimes_{K} V \stackrel{\sim}{\rightarrow} V \otimes_{K} \bar{V}$ qui permute les facteurs induit une isométrie canonique:

$$
\left(N_{K / k}(V), N_{K / k}(\varphi)\right)=\left(N_{K / k}(\bar{V}), N_{K / k}(\bar{\varphi})\right) .
$$

Il résulte en particulier de la propriété (a) que $N_{K / k}(\varphi)$ est une forme quadratique non singulière si $\varphi$ est non singulière (ce que l'on supposera toujours dans la suite). 
Pour décrire une base orthogonale de $N_{K / k}(V)$, posons $K=k(\sqrt{d})$, choisissons $\delta \in K$ tel que $\delta^{2}=d$, et considérons une base orthogonale de $V$ : soit $\left(e_{1}, \ldots, e_{n}\right)$, et soit $\alpha_{i}=$ $\varphi\left(e_{i}\right)(\neq 0)$ pour $i=1, \ldots, n$, c'est-à-dire que $\varphi$ admet la diagonalisation:

$$
\varphi=\left\langle\alpha_{1}, \ldots, \alpha_{n}\right\rangle \text {. }
$$

Pour $i, j=1, \ldots, n$, soit $U_{i j} \subset \bar{V} \otimes_{K} V$ le sous-espace vectoriel sur $K$ engendré par $\overline{e_{i}} \otimes e_{j}$ et $\overline{e_{j}} \otimes e_{i}$. Ce sous-espace est stable sous l'homomorphisme é d'échange; soit $U_{i j}^{\prime} \subset U_{i j}$ l'espace vectoriel sur $k$ des éléments invariants. Comme les sous-espaces $U_{i j}$ sont orthogonaux deux à deux, on a la décomposition en somme directe orthogonale:

$$
N_{K / k}(V)=\underset{1 \leq i \leq j \leq n}{\text { 田 }} U_{i, j}^{\prime}
$$

Pour $i=j$, l'élément $\overline{e_{i}} \otimes e_{i}$ forme une base de $U_{i i}^{\prime}$, et

$$
N_{K / k}(\varphi)\left(\overline{e_{i}} \otimes e_{i}\right)=\overline{\alpha_{i}} \otimes \alpha_{i}=N_{K / k}\left(\alpha_{i}\right) .
$$

Pour $i \neq j$, une base de $U_{i, j}^{\prime}$ est donnée par

$$
\left(\overline{e_{i}} \otimes e_{j}+\overline{e_{j}} \otimes \epsilon_{i}, \overline{e_{i}} \otimes e_{j} \delta-\overline{e_{j}} \otimes e_{i} \delta\right)
$$

Par rapport à cette base, la matrice de la restriction de la forme $N_{K / k}(\varphi)$ est:

$$
\left(\begin{array}{cc}
\overline{\alpha_{i}} \alpha_{j}+\overline{\alpha_{j}} \alpha_{i} & \left(\overline{\alpha_{i}} \alpha_{j}-\overline{\alpha_{j}} \alpha_{i}\right) \delta \\
\left(\overline{\alpha_{i}} \alpha_{j}-\overline{\alpha_{j}} \alpha_{i}\right) \delta & \left(\overline{\alpha_{i}} \alpha_{j}+\overline{\alpha_{j}} \alpha_{i}\right) d
\end{array}\right)
$$

de déterminant $4 N_{K / k}\left(\alpha_{i} \alpha_{j}\right) d$.

1.2 Proposition. Soit $K=k(\sqrt{d})$.

a) Pour toute forme quadratique $\varphi$ de dimension $n$ sur $K$,

$$
\operatorname{disc} N_{K / k}(\varphi)=\operatorname{dét~} N_{K / k}(\varphi)=N_{K / k}(\operatorname{dét} \varphi) \cdot d^{n(n-1) / 2} \cdot k^{\times 2} .
$$

b) Pour le plan hyperbolique $\mathbb{H}_{K}$ sur $K$,

$$
N_{K / k}\left(\mathbb{H}_{K}\right)=\langle 2\rangle \cdot\langle 1,-d\rangle .
$$

c) Pour toute forme quadratique $\varphi$ sur $K$, on a l'égalité suivante dans le groupe de Witt Wk:

$$
N_{K / k}\left(\varphi+\mathbb{H}_{K}\right)=N_{K / k}(\varphi)+N_{K / k}\left(\mathbb{H}_{K}\right) .
$$

Démonstration: Les deux premières parties résultent directement de la décomposition de la norme $N_{K / k}(\varphi)$ associée à une base orthogonale de $\varphi$ : voir (1) ci-dessus. Pour établir la troisième, on forme une base orthogonale $\left(\epsilon_{1}, \ldots, e_{n+2}\right)$ de la somme orthogonale $\varphi \boxplus \mathbb{H}_{K}$ telle que $\left(e_{1}, \ldots, e_{n}\right)$ soit base orthogonale pour $\varphi$ et $\left(e_{n+1}, e_{n+2}\right)$ une base orthogonale de $\mathbb{H}_{K}$. D'après $(1)$, on a

$$
N_{K / k}\left(\varphi \boxplus \mathbb{H}_{K}\right)=N_{K / k}(\varphi) \boxplus N_{K / k}\left(\mathbb{H}_{K}\right) \boxplus\left[\underset{1 \leq i \leq n}{\boxplus}\left(U_{i, n+1}^{\prime} \boxplus U_{i, n+2}^{\prime}\right)\right],
$$

d'où la Proposition car l'espace $U_{i, n+1}^{\prime} \boxplus U_{i, n+2}^{\prime}$ est hyperbolique. 
On se propose à présent de déterminer l'algèbre de Clifford de la forme $N_{K / k}(\varphi)$, dans le cas où la dimension de $\varphi$ est paire.

On note $T_{K / k}$ la forme trace de $K / k$ :

$$
T_{K / k}(x)=x+\bar{x} \quad \text { pour } x \in K .
$$

Pour $x, y \in K^{\times}$on a

$$
T_{K / k}(x) T_{K / k}\left(x y^{2}\right)=T_{K / k}(x y)^{2}+\left[(y-\bar{y}) \delta^{-1}\right]^{2} d N_{K / k}(x),
$$

ce qui montre que si $T_{K / k}(x) T_{K / k}\left(x y^{2}\right) \neq 0$ l'algèbre de quaternions

$$
\left(T_{K / k}(x) T_{K / k}\left(x y^{2}\right),-d N_{K / k}(x)\right)_{k}
$$

est déployée, d'où l'égalité suivante dans le groupe de Brauer $\operatorname{Br} k$ :

$$
\left(T_{K / k}(x),-d N_{K / k}(x)\right)_{k}=\left(T_{K / k}\left(x y^{2}\right),-d N_{K / k}\left(x y^{2}\right)\right)_{k} .
$$

Si $T_{K / k}(x)=0$, alors $x \in \delta \cdot k^{\times}$et par conséquent $-d N_{K / k}(x) \in k^{\times 2}$. Pour tout $y \in K^{\times}$ tel que $T_{K / k}\left(x y^{2}\right) \neq 0$ l'algèbre de quaternions

$$
\left(T_{K / k}\left(x y^{2}\right),-d N_{K / k}\left(x y^{2}\right)\right)_{k}
$$

est alors déployée. Il en résulte que l'on peut définir sans ambiguïté pour toute classe de carrés $\xi \in K^{\times} / K^{\times 2}$ une algèbre de quaternions $\varepsilon(\xi)$ par:

$$
\varepsilon(\xi)=\left(T_{K / k}(x),-d N_{K / k}(x)\right)_{k},
$$

où $x \in K^{\times}$est un représentant quelconque de $\xi$ tel que $T_{K / k}(x) \neq 0$. On a donc $\varepsilon(\xi)=0$ dans $\operatorname{Br} k$ si $\xi$ est représenté par un élément $x \in K^{\times}$tel que $T_{K / k}(x)=0$. D'après la description ci-dessous de la trace d'une forme quadratique, on peut aussi définir $\varepsilon(\xi)$ comme l'algèbre de Clifford de $T_{K / k}(\langle x\rangle)$.

1.3 Proposition. Soit $\varphi$ une forme quadratique de dimension $n=2 m$ sur $K$. L'algèbre de Clifford $C\left(N_{K / k}(\varphi)\right)$ est déterminée dans le groupe de Brauer Br $k$ par la formule suivante:

$$
C\left(N_{K / k}(\varphi)\right)=\varepsilon(\operatorname{disc} \varphi)+\left(d, 2^{m+1}(-1)^{m(m-1) / 2}\right)_{k} .
$$

Démonstration: Si $m=1$, la formule résulte d'un calcul direct. Si $m=2$ et $\operatorname{disc} \varphi=1$, alors

$$
\varphi=\langle a\rangle \otimes \varphi_{1} \otimes \varphi_{2}
$$

pour un certain $a \in K^{\times}$et pour certaines formes quadratiques $\varphi_{1}, \varphi_{2}$ de dimension 2 sur $K$. D'après la propriété $1.1(b)$,

$$
N_{K / k}(\varphi)=\left\langle N_{K / k}(a)\right\rangle \otimes N_{K / k}\left(\varphi_{1}\right) \otimes N_{K / k}\left(\varphi_{2}\right),
$$

d'où

$$
C\left(N_{K / k}(\varphi)\right)=C\left(N_{K / k}\left(\varphi_{1}\right) \otimes N_{K / k}\left(\varphi_{2}\right)\right)
$$

Or, la Proposition 1.2 montre que $N_{K / k}\left(\varphi_{1}\right)$ et $N_{K / k}\left(\varphi_{2}\right)$ sont de discriminant $d$, donc

$$
C\left(N_{K / k}\left(\varphi_{1}\right) \otimes N_{K / k}\left(\varphi_{2}\right)\right)=(d, d)_{k}=(d,-1)_{k} \quad \text { dans } \operatorname{Br} k
$$


Dans le cas général, on procède par induction sur $m(\geq 2)$. Si $\varphi$ est isotrope, il existe une forme quadratique $\psi$ de dimension $2(m-1)$ sur $K$ telle que

$$
\varphi=\psi \boxplus \mathbb{H}_{K} \text {. }
$$

D'après la Proposition $1.2(c)$, on a

$$
N_{K / k}(\varphi)=N_{K / k}(\psi)+N_{K / k}\left(\mathbb{H}_{K}\right) \quad \text { dans } W k,
$$

et la Proposition découle des formules connues pour l'algèbre de Clifford d'une somme directe (voir par exemple [2, p. 121]).

Si $\varphi$ est anisotrope, soit $X$ la quadrique projective d'équation $\varphi=0$ et $R_{K / k}(X)$ la variété algébrique sur $k$ obtenue par restriction des scalaires (à la Weil). Soit encore $k^{\prime}=k\left(R_{K / k}(X)\right)$ et $K^{\prime}=K \otimes_{k} k^{\prime}=K(X \times \bar{X})$. La forme quadratique $\varphi_{K^{\prime}}$ obtenue en étendant les scalaires de $K$ à $K^{\prime}$ est isotrope; comme la Proposition est déjà démontrée pour les formes isotropes, la différence

$$
\Delta=C\left(N_{K / k}(\varphi)\right)-\varepsilon(\operatorname{disc} \varphi)_{k}-\left(d, 2^{m+1}(-1)^{m(m-1) / 2}\right)_{k}
$$

est donc dans le noyau de l'homomorphisme d'extension des scalaires

$$
\mathrm{Br} k \rightarrow \mathrm{Br} k^{\prime}
$$

Ce noyau est déterminé dans [5, Corollary 2.12]: c'est la corestriction de $K$ à $k$ du noyau de l'homomorphisme d'extension des scalaires $\mathrm{Br} K \rightarrow \operatorname{Br} K(X)$. D'après [10, Corollaire 4.3], ce dernier est injectif, sauf si $m=2$ et $\operatorname{disc} \varphi=1$. L'argument donne donc $\Delta=0$ sauf peut-être dans ce cas particulier. La Proposition est ainsi démontrée, car la formule a déjà été établie plus haut dans le cas exceptionnel.

Pour la facilité des comparaisons entre la norme et le transfert, nous terminons cette section en rappelant les principales propriétés du transfert de Scharlau relativement à la forme trace $T_{K / k}$.

Tout espace vectoriel $V$ sur $K$ définit un espace vectoriel $T_{K / k}(V)$ sur $k$, obtenu en ne retenant de $V$ que sa structure d'espace vectoriel sur $k$. Donc, en tant qu'ensembles,

$$
T_{K / k}(V)=V
$$

et l'on a clairement: $T_{K / k}(\bar{V})=T_{K / k}(V)$. Toute forme quadratique $\varphi: V \rightarrow K$ définit une forme quadratique

$$
T_{K / k}(\varphi): T_{K / k}(V) \rightarrow k
$$

par:

$$
T_{K / k}(\varphi)(x)=T_{K / k}(\varphi(x)) \quad \text { pour } x \in V .
$$

Pour souligner l'analogie entre cette construction et celle de la norme $N_{K / k}(\varphi)$, on peut aussi définir $T_{K / k}(V)$ comme l'espace vectoriel sur $k$ des éléments de $\bar{V} \oplus V$ qui sont invariants sous l'homomorphisme d'échange.

$$
\dot{\epsilon}_{\oplus}: \bar{V} \oplus V \rightarrow \bar{V} \oplus V
$$

défini par:

$$
\dot{e}_{\oplus}\left(\overline{v_{1}}, v_{2}\right)=\left(\overline{v_{2}}, v_{1}\right) \quad \text { pour } v_{1}, v_{2} \in V
$$


En effet, les éléments invariants sous $\dot{\epsilon}_{\circledast}$ sont de la forme $(\bar{v}, v)$ avec $v \in V$, et sont donc en bijection naturelle avec $V$ (et donc avec $T_{K / k}(V)$ ). Par cette bijection, la forme quadratique $T_{K / k}(\varphi)$ peut être vue comme induite par la forme quadratique $\bar{\varphi} \boxplus \varphi$ sur $\bar{V} \oplus V$.

1.4 Propriétés. a) Le plongement $T_{K / k}(V) \hookrightarrow \bar{V} \oplus V$ décrit ci-dessus induit une isométrie:

$$
\left(T_{K / k}(V), T_{K / k}(\varphi)\right) \otimes_{k} K=(\bar{V} \oplus V, \bar{\varphi} \boxplus \varphi) .
$$

b) Si $(V, \varphi)$ et $(W, \psi)$ sont des espaces quadratiques sur $K$, il y a une isométrie canonique:

$$
\left(T_{K / k}(V \oplus W), T_{K / k}(\varphi \boxplus \psi)\right)=\left(T_{K / k}(V), T_{K / k}(\varphi)\right) \boxplus\left(T_{K / k}(W), T_{K / k}(\psi)\right) .
$$

c) Pour tout espace quadratique $(V, \varphi)$ sur $K$, il y a une isométrie canonique:

$$
\left(T_{K / k}(V), T_{K / k}(\varphi)\right)=\left(T_{K / k}(\bar{V}), T_{K / k}(\bar{\varphi})\right) .
$$

(Voir [2, Chap. 7], [8, Chap. 2, §8]).

Comme précédemment, posons $K=k(\sqrt{d})$ et choisissons $\delta \in K$ tel que $\delta^{2}=d$. Soit $\left(e_{1}, \ldots, e_{n}\right)$ une base othogonale de $V$ et soit

$$
\varphi=\left\langle\alpha_{1}, \ldots, \alpha_{n}\right\rangle
$$

la diagonalisation correspondante. On a alors une décomposition de $T_{K / k}(V)$ en somme orthogonale:

$$
T_{K / k}(V)=\stackrel{n}{\stackrel{n}{⿴ 囗 十 1}} V_{i}
$$

où $V_{i}=T_{K / k}\left(e_{i} \cdot K\right)$ est le sous-espace engendré par $e_{i}$ et $e_{i} \delta$. Par rapport à cette base, la matrice de la restriction de $T_{K / k}(\varphi)$ est

$$
\left(\begin{array}{cc}
T_{K / k}\left(\alpha_{i}\right) & T_{K / k}\left(\alpha_{i} \delta\right) \\
T_{K / k}\left(\alpha_{i} \delta\right) & d T_{K / k}\left(\alpha_{i}\right)
\end{array}\right)
$$

de déterminant $4 d N_{K / k}\left(\alpha_{i}\right)$. La Proposition suivante se déduit de cette décomposition par des calculs directs:

1.5 Proposition. Soit $K=k(\sqrt{d})$.

a) Pour toute forme quadratique $\varphi$ de dimension $n$ sur $K$,

$$
\begin{aligned}
\operatorname{dét} T_{K / k}(\varphi) & =N_{K / k}(\operatorname{dét} \varphi) \cdot d^{n} \cdot k^{\times 2}, \\
\operatorname{disc} T_{K / k}(\varphi) & =(-1)^{n} N_{K / k}(\operatorname{disc} \varphi) \cdot d^{n} \cdot k^{\times 2} .
\end{aligned}
$$

b) Pour toute forme quadratique $\varphi$ sur $k$,

$$
T_{K / k}\left(\varphi_{K}\right)=\varphi \cdot\langle 2\rangle \cdot\langle 1, d\rangle=\varphi \cdot T_{K / k}\left(\langle 1\rangle_{K}\right) .
$$

c) Pour le plan hyperbolique $\mathbb{H}_{K}$ sur $K$,

$$
T_{K / k}\left(\mathbb{H}_{K}\right)=2 \mathbb{H}_{k} .
$$


d) La trace $T_{K / k}$ induit un homomorphisme de groupes de Witt:

$$
\left(T_{K / k}\right)_{*}: W K \rightarrow W k
$$

Il y a également une relation directe entre l'algèbre de Clifford de la forme $T_{K / k}(\varphi)$ et celle de $\varphi$, qui s'exprime à l'aide de la corestriction

$$
\operatorname{Cor}_{K / k}: \mathrm{Br} K \rightarrow \mathrm{Br} k .
$$

Soit $I K$ l'idéal fondamental de $W K$, dont les éléments sont représentés par les formes quadratiques de dimension paire. L'application qui à toute forme quadratique associe son algèbre de Clifford induit un homomorphisme

$$
C: I^{2} K \rightarrow \operatorname{Br} K \text {. }
$$

Le résultat suivant est dû̀ Arason [1, Satz 4.18] (voir aussi [6, §6, Cor.1]):

1.6 Lemme. Le diagramme

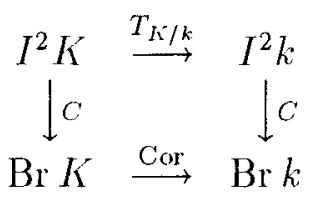

est commutatif.

Démonstration: On commence par mettre en évidence un système de générateurs de $I^{2} K$ modulo $I^{3} K$. L'idéal $I^{2} K$ est engendré par les formes de Pfister doubles, c'est-à-dire les formes quadratiques

$$
\langle\langle a, b\rangle\rangle=\langle 1,-a\rangle \otimes\langle 1,-b\rangle \quad \text { avec } a, b \in K^{\times} .
$$

Comme pour $a, b, c \in K^{\times}$,

$$
\langle\langle a, b\rangle\rangle-\langle c\rangle \cdot\langle\langle a, b\rangle\rangle \in I^{3} K,
$$

il en résulte que toute forme $\varphi \in I^{2} K$ est somme dans $W K$ de formes de Pfister doubles et d'une forme de $I^{3} K$ :

$$
\varphi \equiv \sum_{i}\left\langle\left\langle a_{i}, b_{i}\right\rangle\right\rangle \bmod I^{3} K
$$

pour certains $a_{i}, b_{i} \in K^{\times}$.

Pour $a, b \in K^{\times}$, on peut trouver $\alpha, \beta \in k^{\times}$tels que

$$
\alpha a+\beta b=0 \text { ou } 1,
$$

vu que $[K: k]=2$. La forme $\langle\langle\alpha a, \beta b\rangle\rangle$ est alors hyperbolique, donc

$$
\langle\langle a, b\rangle\rangle+\langle\langle\alpha, b\rangle\rangle+\langle\langle a, \beta\rangle\rangle+\langle\langle\alpha, \beta\rangle\rangle \equiv\langle\langle\alpha a, \beta b\rangle\rangle=0 \bmod I^{3} K .
$$

On peut donc remplacer dans la décomposition (2) ci-dessus chacune des formes $\left\langle\left\langle a_{i}, b_{i}\right\rangle\right\rangle$ par une somme de formes de Pfister doubles du type $\langle\langle\alpha, b\rangle\rangle$ avec $\alpha \in k^{\times}$et $b \in K^{\times}$. Comme l'algèbre de Clifford de toute forme de $I^{3} K$ est déployée, il suffit de prouver:

$$
C\left(T_{K / k}\langle\langle\alpha, b\rangle\rangle\right)=\operatorname{Cor}_{K / k}(C\langle\langle\alpha, b\rangle\rangle) \quad \text { pour } \alpha \in k^{\times} \text {et } b \in K^{\times},
$$

ce qui résulte d'un calcul direct utilisant la formule de projection. 
1.7 Proposition. Pour toute forme quadratique $\varphi$ de dimension paire sur $K$,

$$
C\left(T_{K / k}(\varphi)\right)=\operatorname{Cor}_{K / k}(C(\varphi))+\varepsilon(\operatorname{disc} \varphi)+\left(d, 2 N_{K / k}(\operatorname{disc} \varphi)\right)_{k} \quad \text { dans } \operatorname{Br} k \text {. }
$$

Démonstration: Comme la dimension de $\varphi$ est paire, on a

$$
\varphi \equiv\langle\langle\operatorname{disc} \varphi\rangle\rangle \bmod I^{2} K
$$

Du Lemme précédent, on déduit alors:

$$
C\left(T_{K / k}(\varphi-\langle\langle\operatorname{disc} \varphi\rangle\rangle)\right)=\operatorname{Cor}_{K / k}(C(\varphi-\langle\langle\operatorname{disc} \varphi\rangle\rangle)) .
$$

La Proposition s'en déduit par l'additivité de la trace (propriété 1.4(b)) et celle de l'algèbre de Clifford, suivant laquelle

$$
C(\varphi \boxplus \psi)=C(\varphi)+C(\psi)+(\operatorname{disc} \varphi, \operatorname{disc} \psi)_{k} \quad \text { dans } \operatorname{Br} k
$$

pour $\varphi, \psi$ de dimension paire (voir $[2$, p. 121]).

Signalons le cas particulier suivant, obtenu en prenant $\varphi=\langle a, b\rangle$ (ou $\varphi=\langle\langle a, b\rangle\rangle)$ :

1.8 Corollaire. Pour $a, b \in K^{\times}$,

$$
\operatorname{Cor}_{K / k}(a, b)_{K}=\varepsilon(1)+\varepsilon(a)+\varepsilon(b)+\varepsilon(a b)+\left(N_{K / k}(a), N_{K / k}(b)\right)_{k}
$$

\section{La norme et la trace des espaces hermitiens}

Comme dans la section précédente, $K / k$ est une extension quadratique de corps et $V$ un espace vectoriel sur $K$. Au lieu d'une forme quadratique, on considère cette fois une forme hermitienne

$$
h: V \times V \rightarrow K
$$

relativement à l'automorphisme non trivial ${ }^{-}$de $K / k$. On a donc

$$
h(v \alpha, w \beta)=\bar{\alpha} h(v, w) \beta \quad \text { et } \quad h(w, v)=\overline{h(v, w)} \quad \text { pour } v, w \in V \text { et } \alpha, \beta \in K .
$$

Le produit $\bar{h} \otimes h$ est maintenant une forme hermitienne sur $\bar{V} \otimes_{K} V$, définie par

$$
\bar{h} \otimes h\left(\overline{v_{1}} \otimes v_{2}, \overline{w_{1}} \otimes w_{2}\right)=\overline{h\left(v_{1}, v_{2}\right)} h\left(w_{1}, w_{2}\right) \quad \text { pour } v_{1}, v_{2}, w_{1}, w_{2} \in V .
$$

Cette forme hermitienne satisfait la propriété suivante vis-à-vis de l'homomorphisme $e ́$ d'échange:

$$
\bar{h} \otimes h(\dot{e}(x), \dot{e}(y))=\overline{\bar{h} \otimes h(x, y)} \quad \text { pour } x, y \in \bar{V} \otimes_{K} V .
$$

Dès lors, la restriction de $\bar{h} \otimes h$ à la norme $N_{K / k}(V)$ est une forme bilinéaire symétrique, que l'on note $N_{K / k}(h)$. Abusant quelque peu des notations, on notera de la même manière la forme quadratique associée. On a donc

$$
N_{K / k}(h)(\bar{v} \otimes w+\bar{w} \otimes v)=2 h(v, v) h(w, w)+T_{K / k}\left(h(v, w)^{2}\right) .
$$


2.1 Propriétés. a) Si $(V, h)$ et $\left(V^{\prime}, h^{\prime}\right)$ sont des espaces hermitiens sur $K$, il y a une isométrie canonique:

$$
\left(N_{K / k}\left(V \otimes_{K} V^{\prime}\right), N_{K / k}\left(h \otimes h^{\prime}\right)\right)=\left(N_{K / k}(V), N_{K / k}(h)\right) \otimes_{k}\left(N_{K / k}\left(V^{\prime}\right), N_{K / k}\left(h^{\prime}\right)\right) .
$$

b) L'isomorphisme canonique $\bar{V} \otimes_{K} V \stackrel{\sim}{\rightarrow} V \otimes_{K} \bar{V}$ qui permute les facteurs induit une isométrie canonique:

$$
\left(N_{K / k}(V), N_{K / k}(h)\right)=\left(N_{K / k}(\bar{V}), N_{K / k}(\bar{h})\right) .
$$

Comme précédemment, on peut décrire une diagonalisation de la forme $N_{K / k}(h)$ au moyen d'une base orthogonale de $V$ : soit $\left(e_{1}, \ldots, e_{n}\right)$ une telle base et soit

$$
h\left(e_{i}, e_{i}\right)=\alpha_{i}(\in k) .
$$

Posons $K=k(\sqrt{d})$ et choisissons $\delta \in K$ tel que $\delta^{2}=d$. Les éléments

$$
\left(\overline{e_{i}} \otimes e_{i}\right)_{1 \leq i \leq n},\left(\overline{e_{i}} \otimes e_{j}+\overline{e_{j}} \otimes e_{i}\right)_{1 \leq i<j \leq n} \quad \text { et } \quad\left(\overline{e_{i}} \otimes e_{j} \delta-\overline{e_{j}} \otimes e_{i} \delta\right)_{1 \leq i<j \leq n}
$$

forment alors une base orthogonale de $N_{K / k}(V)$ pour la forme $N_{K / k}(h)$. Par rapport à cette base, la forme $N_{K / k}(h)$ admet la diagonalisation suivante:

$$
N_{K / k}(h)=n\langle 1\rangle \boxplus\langle 2\rangle \cdot\langle 1,-d\rangle \cdot\left(\underset{1 \leq i<j \leq n}{\mathbb{M}_{j<n}}\left\langle\alpha_{i} \alpha_{j}\right\rangle\right) .
$$

En particulier, la forme $N_{K / k}(h)$ est non singulière si $h$ est non singulière (c'est-à-dire que $\alpha_{i} \neq 0$ pour tout $i$ ), ce que l'on supposera toujours dans la suite. En utilisant la diagonalisation ci-dessus, il est facile de calculer le discriminant de $N_{K / k}(h)$ :

$$
\operatorname{disc} N_{K / k}(h)=\operatorname{dét} N_{K / k}(h)=(-d)^{n(n-1) / 2} \cdot k^{2},
$$

où $n=\operatorname{dim}_{K} V$.

Le déterminant de la matrice de $h$ par rapport à une base quelconque de $V$ est un élément de $k^{\times}$que tout changement de base multiplie par une norme de $K^{\times}$. Le déterminant de $h$ est donc bien défini dans le quotient $k^{\times} / N_{K / k}\left(K^{\times}\right)$, et l'on peut définir un invariant analogue au discriminant, représenté par une algèbre de quaternions:

$$
\operatorname{disc}(h)=\left(d,(-1)^{n(n-1) / 2} \operatorname{dét} h\right)_{k} \in \operatorname{Br} k,
$$

où $n=\operatorname{dim}_{K} V$. Cet invariant est en relation directe avec l'algèbre de Clifford de la forme norme:

2.2 Proposition. Si $\operatorname{dim}_{K} V=n=2 m$,

$$
C\left(N_{K / k}(h)\right)=\operatorname{disc}(h)+\left(-d, 2^{m}(-1)^{m(m-1) / 2}\right)_{k} \quad \text { dans } \operatorname{Br} k .
$$

Cette Proposition s'obtient par les formules qui décrivent l'algèbre de Clifford d'une somme directe (voir [2, p. 121]), en utilisant une diagonalisation de la forme $N_{K / k}(h)$. 
La norme $N_{K / k}(h)$ admet aussi une version additive: sur le $k$-espace vectoriel $T_{K / k}(V)$, on définit une forme quadratique $T_{K / k}(h)$ par:

$$
T_{K / k}(h)(x)=T_{K / k}(h(x, x))=2 h(x, x) \quad \text { pour } x \in V .
$$

Si $\left(e_{1}, \ldots, e_{n}\right)$ est une base orthogonale de $V$ sur $K$ pour la forme hermitienne $h$, alors $\left(e_{1}, e_{1} \delta, \ldots, e_{n}, e_{n} \delta\right)$ est une base orthogonale de $V$ sur $k$ pour la forme $T_{K / k}(h)$. En posant $\alpha_{i}=h\left(e_{i}, e_{i}\right)$ pour $i=1, \ldots, n$, on obtient

$$
T_{K / k}(h)=\langle 2\rangle \cdot\langle 1,-d\rangle \cdot\left\langle\alpha_{1}, \ldots, \alpha_{n}\right\rangle
$$

Le déterminant et l'algèbre de Clifford de la forme $T_{K / k}(h)$ sont dès lors faciles à calculer:

2.3 Proposition. Avec les notations ci-dessus,

$$
\begin{aligned}
\operatorname{dét} T_{K / k}(h) & =(-d)^{n} \cdot k^{\times 2}, \\
\operatorname{disc} T_{K / k}(h) & =d^{n} \cdot k^{\times 2}
\end{aligned}
$$

et, si $n$ est pair,

$$
C\left(T_{K / k}(h)\right)=\operatorname{disc}(h) \quad \text { dans } \mathrm{Br} k \text {. }
$$

\section{La forme trace des algèbres simples centrales}

Pour toute algèbre simple centrale $A$ de dimension finie sur un corps $K$, on note

$$
\operatorname{deg} A=\sqrt{\operatorname{dim}_{K} A}
$$

le degré de $A$ et

$$
T_{A}: A \rightarrow K
$$

la forme quadratique définie par

$$
T_{A}(x)=\operatorname{Trd}_{A}\left(x^{2}\right) \quad \text { pour } x \in A,
$$

où $\operatorname{Trd}_{A}$ est la trace réduite. Cette forme a été étudiée en particulier par Lewis [3] et par Lewis-Morales [4]. Les propriétés suivantes ont déjà été mises en évidence dans [3]:

3.1 Propriétés. a) Si $A$ est déployée par une extension de $K$ de degré impair (par exemple si $A$ est déployée ou si le degré de A est impair),

$$
T_{A}=n\langle 1\rangle \boxplus \frac{n(n-1)}{2} \mathbb{H}_{K},
$$

où $n=\operatorname{deg} A$.

b) Si $A$ est une algèbre simple centrale de degré $n$,

$$
\operatorname{disc} T_{A}=\operatorname{dét} T_{A}=(-1)^{n(n-1) / 2} \text {. }
$$

c) Si $A$ et $A^{\prime}$ sont deux algèbres simples centrales sur $K$,

$$
T_{A \otimes K} A^{\prime}=T_{A} \otimes T_{A^{\prime}} .
$$


Démonstration: (a) Si $A$ est une algèbre de matrices, la forme $T_{A}$ est facile à déterminer par un calcul direct. Si $A$ est déployée par une extension $L / K$ de degré impair, alors la différence $T_{A}-n\langle 1\rangle$ est dans le noyau de l'homomorphisme d'extension des scalaires $W K \rightarrow W L$. Or un théorème de Springer [2, p. 198], [8, p. 46] montre que cet homomorphisme est injectif. La propriété (a) est donc démontrée.

(b) Soit $F / K$ une extension qui déploie $A$ et telle que $K$ soit algébriquement clos dans $F$. (On peut prendre par exemple pour $F$ le corps des fonctions de la variété de Severi-Brauer de $A$ ). L'homomorphisme $K^{\times} / K^{\times 2} \rightarrow F^{\times} / F^{\times 2}$ induit par l'inclusion $K \subset F$ est alors injectif. Or, comme $F$ déploie $A$, le quotient $\operatorname{disc} T_{A} \cdot \operatorname{disc}\left(n\langle 1\rangle \boxplus \frac{n(n-1)}{2} \mathbb{H}\right)^{-1}$ est dans le noyau de cet homomorphisme. La propriété (b) en découle.

Enfin, (c) provient de la formule suivante:

$$
\operatorname{Trd}_{A \otimes_{K} A^{\prime}}\left(a \otimes a^{\prime}\right)=\operatorname{Trd}_{A}(a) \cdot \operatorname{Trd}_{A^{\prime}}\left(a^{\prime}\right) \quad \text { pour } a \in A \text { et } a^{\prime} \in A^{\prime} .
$$

On se propose d'étudier diverses constructions liées à la forme $T_{A}$.

Supposons pour commencer que $A$ admette une involution de seconde espèce $\sigma$, c'est-àdire un anti-automorphisme d'ordre 2 dont la restriction au centre $K$ n'est pas l'identité. Le sous-corps $k \subset K$ des éléments invariants sous $\sigma$ est alors de codimension 2. Soit $(A, \sigma)_{+}$l'espace vectoriel sur $k$ des éléments de $A$ symétriques (c'est-à-dire invariants) sous $\sigma$. La restriction de $T_{A}$ à $(A, \sigma)_{+}$est une forme quadratique

$$
t:(A, \sigma)_{+} \rightarrow k .
$$

Exemple 1: Soit $V$ un espace vectoriel sur un corps $K$, extension quadratique d'un souscorps $k$, muni d'une forme hermitienne $h$ non singulière. L'algèbre $A=\operatorname{End}_{K}(V)$ admet alors une involution $\sigma_{h}$ de seconde espèce adjointe à $h$, définie par la relation:

$$
h(x, f(y))=h\left(\sigma_{h}(f)(x), y\right) \quad \text { pour } x, y \in V \text { et } f \in A .
$$

3.2 Proposition. Il y a une isométrie naturelle:

$$
\left(\left(\operatorname{End}_{K}(V), \sigma_{h}\right)_{+}, t\right) \simeq\left(N_{K / k}(\bar{V}), N_{K / k}(\bar{h})\right) \quad\left(=\left(N_{K / k}(V), N_{K / k}(h)\right)\right) .
$$

Démonstration: La forme hermitienne $h$, étant supposée non singulière, induit un isomorphisme

$$
\hat{h}: \bar{V} \stackrel{\sim}{\rightarrow} V^{*}=\operatorname{Hom}_{K}(V, K)
$$

défini par:

$$
\hat{h}(\bar{x})(y)=h(x, y) \quad \text { pour } x, y \in V .
$$

En composant cet isomorphisme avec l'isomorphisme canonique $\operatorname{End}_{K}(V)=V \otimes_{K} V^{*}$, on obtient un isomorphisme canonique d'espaces vectoriels sur $K$ :

$$
\operatorname{End}_{K}(V)=V \otimes_{K} \bar{V},
$$

qui transporte l'involution $\sigma_{h}$ sur l'homomorphisme é d'échange et induit par conséquent un isomorphisme canonique:

$$
\left(\operatorname{End}_{K}(V), \sigma_{h}\right)_{+}=N_{K / k}(\bar{V}) .
$$


On vérifie de plus:

$$
\operatorname{Trd}_{A}\left(\sigma_{h}(f) g\right)=h \otimes \bar{h}(f, g) \quad \text { pour } f, g \in \operatorname{End}_{K}(V)
$$

donc l'isomorphisme canonique $\operatorname{End}_{K}(V)=V \otimes_{K} \bar{V}$ induit l'isométrie annoncée, par restriction aux éléments invariants sous $\sigma_{h}$ d'une part et sous é d'autre part.

Exemple 2: Considérons une algèbre simple centrale $A$ quelconque sur $K$. On note $\bar{A}$ l'algèbre conjuguée, définie par:

$$
\bar{A}=\{\bar{a} \mid a \in A\}
$$

avec les opérations:

$$
\bar{a}+\bar{b}=\overline{a+b} \quad \bar{a} \bar{b}=\overline{a b} \quad \bar{a} \alpha=\overline{a \bar{\alpha}} \quad \text { pour } a, b \in A \text { et } \alpha \in K .
$$

Soit $A^{\text {op }}$ l'algèbre opposée de $A$. Le produit $\bar{A} \otimes_{K} A^{\text {op }}$ porte une involution canonique $\theta$ de deuxième espèce, définie par

$$
\theta\left(\bar{a} \otimes b^{\mathrm{op}}\right)=\bar{b} \otimes a^{\mathrm{op}} \quad \text { pour } a, b \in A .
$$

3.3 Proposition. L'application canonique $\bar{A} \otimes_{K} A \rightarrow \bar{A} \otimes_{K} A^{\mathrm{op}}$ qui envoie $\bar{a} \otimes b$ sur $\bar{a} \otimes b^{\mathrm{op}}$ induit une isométrie d'espaces quadratiques sur $k$ :

$$
\left(N_{K / k}(A), N_{K / k}\left(T_{A}\right)\right)=\left(\left(\bar{A} \otimes_{K} A^{\mathrm{op}}, \theta\right)_{+}, t\right) .
$$

Démonstration: L'application canonique $\bar{A} \otimes A \rightarrow \bar{A} \otimes A^{\mathrm{op}}$ transporte l'homomorphisme $e$ d'échange sur l'involution $\theta$; elle fait donc se correspondre les espaces d'éléments invariants:

$$
N_{K / k}(A) \stackrel{\sim}{\rightarrow}\left(\bar{A} \otimes_{K} A^{\mathrm{op}}, \theta\right)_{+} .
$$

Pour voir que cette correspondance est une isométrie, il suffit de remarquer:

$$
\overline{T_{A}} \otimes T_{A}=T_{\bar{A}} \otimes T_{A^{\mathrm{op}}}=T_{\bar{A} \otimes A^{\mathrm{op}}}
$$

Les deux exemples précédents se présentent dans la situation suivante:

Soit $A$ une algèbre simple centrale sur un corps $k$. Supposons que $A$ contienne un corps $K$, extension quadratique de $k$. Son degré est alors divisible par 2 ; soit

$$
\operatorname{deg} A=n=2 m
$$

Comme précédemment, on note $K=k(\sqrt{d})$ et on choisit $\delta \in K$ tel que $\delta^{2}=d$. Soit $B$ le centralisateur de $K$ dans $A$ - l'algèbre $B$ est donc simple centrale sur $K$ de degré $m$ - et soit

$$
B^{\prime}=\{x \in A \mid x \delta=-\delta x\} .
$$

L'ensemble $B^{\prime}$ est de manière naturelle un espace vectoriel sur $K$, la multiplication scalaire * étant définie comme la multiplication dans $A$, le facteur de $K$ étant placé à droite: pour $b^{\prime} \in B^{\prime}$ et $\alpha \in K$ on pose

$$
b^{\prime} * \alpha=b^{\prime} \alpha=\bar{\alpha} b^{\prime} .
$$


3.4 Lemme. En tant qu'espace quadratique sur $k$ pour la forme $T_{A}$, l'algèbre $A$ se décompose en somme directe orthogonale:

$$
A=B \boxplus B^{\prime}
$$

et

$$
\operatorname{dim} B=\operatorname{dim} B^{\prime}=\frac{1}{2} \operatorname{dim} A=2 m^{2} .
$$

Démonstration: Tout élément $a \in A$ admet une décomposition unique:

$$
a=b+b^{\prime}
$$

avec $b=\frac{1}{2}\left(a+\delta a \delta^{-1}\right) \in B$ et $b^{\prime}=\frac{1}{2}\left(a-\delta a \delta^{-1}\right) \in B^{\prime}$. Tout élément $b^{\prime} \in B^{\prime}$ est de trace réduite nulle, car

$$
\operatorname{Trd}_{A}\left(b^{\prime}\right)=\operatorname{Trd}_{A}\left(\delta b^{\prime} \delta^{-1}\right)=-\operatorname{Trd}_{A}\left(b^{\prime}\right) .
$$

Pour $b \in B$ et $b^{\prime} \in B^{\prime}$, on a $b b^{\prime} \in B^{\prime}$, donc par l'observation précédente:

$$
\operatorname{Trd}_{A}\left(b b^{\prime}\right)=0
$$

Cela prouve que $B$ et $B^{\prime}$ sont orthogonaux pour la forme $T_{A}$. Comme $\operatorname{deg} B=m$, on a $\operatorname{dim}_{k} B=2 m^{2}$, donc $\operatorname{dim}_{k} B^{\prime}=2 m^{2}$.

Soit $T^{\prime}$ la restriction de la forme quadratique $T_{A}$ au sous-espace $B^{\prime} \subset A$. Comme le produit de deux éléments de $B^{\prime}$ est dans $B$, on peut définir une forme hermitienne

$$
h: B^{\prime} \times B^{\prime} \rightarrow K
$$

par:

$$
h\left(b_{1}^{\prime}, b_{2}^{\prime}\right)=\operatorname{Trd}_{B}\left(b_{1}^{\prime} b_{2}^{\prime}\right)
$$

Pour $b^{\prime} \in B^{\prime}$, on a

$$
T^{\prime}\left(b^{\prime}\right)=\operatorname{Trd}_{A}\left(b^{\prime 2}\right)=T_{K / k}\left(\operatorname{Trd}_{B}\left(b^{\prime 2}\right)\right)=T_{K / k}\left(h\left(b^{\prime}, b^{\prime}\right)\right),
$$

donc $T^{\prime}=T_{K / k}(h)$.

3.5 Proposition. Il y a une isométric naturelle:

$$
\left(N_{K / k}\left(B^{\prime}\right), N_{K / k}(h)\right) \simeq\left(N_{K / k}(B), N_{K / k}\left(T_{B}\right)\right)
$$

Démonstration: La multiplication dans $A$ induit un homomorphisme d'algèbres sur $K$ :

$$
\Phi: \bar{B} \otimes_{K} B^{\mathrm{op}} \rightarrow \operatorname{End}_{K}\left(B^{\prime}\right)
$$

défini par:

$$
\Phi\left(\overline{b_{1}} \otimes b_{2}^{\mathrm{oP}}\right)\left(b^{\prime}\right)=b_{1} b^{\prime} b_{2} .
$$

Comme l'algèbre $\bar{B} \otimes_{K} B^{\text {op }}$ est simple, l'homomorphisme $\Phi$ est injectif. Comme de plus $\operatorname{dim}_{k} B^{\prime}=\operatorname{dim}_{k} B$, l'homomorphisme $\Phi$ est un isomorphisme. Pour $b_{1}, b_{2} \in B$ et $b_{1}^{\prime}, b_{2}^{\prime} \in$ $B^{\prime}$ on a

$$
h\left(b_{1}^{\prime}, b_{1} b_{2}^{\prime} b_{2}\right)=\operatorname{Trd}_{B}\left(b_{1}^{\prime} b_{1} b_{2}^{\prime} b_{2}\right)=\operatorname{Trd}_{B}\left(b_{2} b_{1}^{\prime} b_{1} b_{2}^{\prime}\right)=h\left(b_{2} b_{1}^{\prime} b_{1}, b_{2}^{\prime}\right)
$$


Dès lors, l'isomorphisme $\Phi$ transporte l'involution canonique $\theta$ de $\bar{B} \otimes_{K} B^{\text {op }}$ sur l'involution $\sigma_{h}$ adjointe à la forme hermitienne $h$ :

$$
\Phi:\left(\bar{B} \otimes_{K} B^{o P}, \theta\right) \stackrel{\sim}{\rightarrow}\left(\operatorname{End}_{K}\left(B^{\prime}\right), \sigma_{h}\right) .
$$

Par les Propositions 3.2 et 3.3, on en déduit les isométries suivantes:

$$
\begin{aligned}
\left(N_{K / k}\left(B^{\prime}\right), N_{K / k}(h)\right) & \simeq\left(\left(\operatorname{End}_{K}\left(B^{\prime}\right), \sigma_{h}\right)_{+}, t\right) \\
& \simeq\left(\left(\bar{B} \otimes K B^{\mathrm{op}}, \theta\right)_{+}, t\right) \\
& \simeq\left(N_{K / k}(B), N_{K / k}\left(T_{B}\right)\right) .
\end{aligned}
$$

Comme application des résultats précédents, nous retrouvons la formule qui donne l'algèbre de Clifford de la forme $T_{A}$. Vu la propriété 3.1(a), il suffit de considérer le cas où le degré de $A$ est pair. Pour tout entier pair $n>1$, on note $C(n)$ l'algèbre de Clifford de la forme $n\langle 1\rangle$. On a donc:

$$
C(n)=\frac{1}{2} \frac{n}{2}\left(\frac{n}{2}-1\right) \cdot(-1,-1)_{k} \quad \text { dans } \operatorname{Br} k .
$$

3.6 Proposition. Pour toute algèbre simple centrale $A$ de degré $n$ pair sur un corps $k$, on $a$

$$
C\left(T_{A}\right)=\frac{n}{2} \cdot A+C(n) \quad \text { dans } \operatorname{Br} k .
$$

Cette formule a été établie indépendamment par Saltman [7], Serre [9, p. 118] et par Lewis-Morales [4]. La démonstration qui suit utilise une méthode différente.

Démonstration: Supposons d'abord que $A$ soit une algèbre à division dont le degré est une puissance de 2: soit

$$
n=2^{\ell}, \quad \text { avec } \ell \geq 1 \text {. }
$$

Si $\ell=1$, alors l'algèbre $A$ est une algèbre de quaternions, et la Proposition est facile à établir par un calcul direct. Pour la suite de la démonstration, on peut donc supposer $\ell \geq 2$ et raisonner par induction sur $\ell$.

Soit $M \subset A$ un sous-corps maximal. On considère une clôture galoisienne $\bar{M}$ de $M / k$ et $L \subset \bar{M}$ le sous-corps des invariants sous un 2-sous-groupe de Sylow du groupe de Galois de $\bar{M} / k$. Le composé $L \cdot M \subset \bar{M}$ contient alors une extension quadratique de $L$. De plus, l'extension $L / k$ est de degré impair, donc l'homomorphisme d'extension des scalaires $\mathrm{Br} k \rightarrow \mathrm{Br} L$ est injectif sur les éléments de 2-torsion. Dès lors, il suffit de démontrer la Proposition après extension des scalaires à $L$; quitte à remplacer $A$ par $A \otimes_{k} L$, on peut donc supposer que $A$ contient un corps $K$ extension quadratique de $k$.

On reprend alors les notations du Lemme 3.4:

$$
A=B \boxplus B^{\prime} .
$$

Pour $b \in B$,

$$
\operatorname{Trd}_{A}(b)=T_{K / k}\left(\operatorname{Trd}_{B}(b)\right),
$$

donc la restriction à $B$ de la forme $T_{A}$ est la trace de la forme $T_{B}$ :

$$
\left.T_{A}\right|_{B}=T_{K / k}\left(T_{B}\right)
$$


Comme $\operatorname{disc} T_{B}= \pm 1$, la Proposition 1.5(a) donne:

$$
\left.\operatorname{disc} T_{A}\right|_{B}=1 \text {, }
$$

donc la décomposition (3) entraîne:

$$
C\left(T_{A}\right)=C\left(\left.T_{A}\right|_{B}\right)+C\left(T^{\prime}\right) \quad \text { dans } \operatorname{Br} k .
$$

On calcule alors séparément les deux termes du membre de droite.

Vu que $\left.T_{A}\right|_{B}=T_{K / k}\left(T_{B}\right)$ et $N_{K / k}\left(\operatorname{disc} T_{B}\right)=1$, on déduit de la Proposition 1.7:

$$
C\left(\left.T_{A}\right|_{B}\right)=\operatorname{Cor}_{K / k}\left(C\left(T_{B}\right)\right)+\varepsilon\left(\operatorname{disc} T_{B}\right)+(d, 2)_{k} .
$$

Par hypothèse d'induction,

$$
C\left(T_{B}\right)=2^{\ell-2} \cdot B+C\left(2^{\ell-1}\right) \quad \text { dans } \operatorname{Br} K
$$

par ailleurs, comme $B$ est le centralisateur de $K$ dans $A$, on a $A \otimes K=B$ dans $\operatorname{Br} K$, donc

$$
\operatorname{Cor}_{K / k}(B)=\operatorname{Cor}_{K / k}\left(A \otimes_{k} K\right)=2 \cdot A \quad \text { dans } \operatorname{Br} k .
$$

Par conséquent,

$$
\operatorname{Cor}_{K / k}\left(C\left(T_{B}\right)\right)=2^{\ell-1} \cdot A \quad \text { dans } \operatorname{Br} k
$$

et (5) donne:

$$
C\left(\left.T_{A}\right|_{B}\right)=2^{\ell-1} \cdot A+\varepsilon\left(\operatorname{disc} T_{B}\right)+(d, 2)_{k} .
$$

Pour le calcul de $C\left(T^{\prime}\right)$, on utilise la relation $T^{\prime}=T_{K / k}(h)$. Celle-ci entraîne, par la Proposition 2.3:

$$
C\left(T^{\prime}\right)=\operatorname{disc}(h) \quad \text { dans } \operatorname{Br} k .
$$

La Proposition 2.2 donne par ailleurs la relation suivante dans $\operatorname{Br} k$ :

$$
C\left(N_{K / k}(h)\right)=\operatorname{disc}(h)+\left\{\begin{array}{cl}
(-d,-1)_{k} & \text { si } \ell=2 \\
0 & \text { si } \ell>2 .
\end{array}\right.
$$

Or, la Proposition 3.5 donne une isométrie:

$$
\left(N_{K / k}\left(B^{\prime}\right), N_{K / k}(h)\right) \simeq\left(N_{K / k}(B), N_{K / k}\left(T_{B}\right)\right),
$$

donc en combinant les relations précédentes on trouve:

$$
C\left(T^{\prime}\right)=C\left(N_{K / k}\left(T_{B}\right)\right)+\left\{\begin{array}{cl}
(-d,-1)_{k} & \text { si } \ell=2 \\
0 & \text { si } \ell>2
\end{array} \quad \text { dans } \operatorname{Br} k .\right.
$$

On utilise alors la formule qui donne l'algèbre de Clifford de la norme d'un espace quadratique (Proposition 1.3) pour obtenir:

$$
C\left(T^{\prime}\right)=\varepsilon\left(\operatorname{disc} T_{B}\right)+(d, 2)_{k}+\left\{\begin{array}{cc}
(-1,-1)_{k} & \text { si } \ell=2 \\
0 & \text { si } \ell>2 .
\end{array}\right.
$$

Les relations (4), (6) et (7) achèvent la démonstration dans le cas où $A$ est une algèbre à division dont le degré est une puissance de 2 . 
Le cas général se ramène au cas particulier étudié ci-dessus par les arguments suivants: d'après la "décomposition primaire" des algèbres simples centrales et le théorème de Wedderburn, toute algèbre simple centrale $A$ admet une décomposition:

$$
A=D \otimes_{k} E
$$

où $D$ est une algèbre à division dont le degré est une puissance de 2 et $E$ est une algèbre simple centrale sur $k$ déployée par une extension de degré impair. Soit

$$
\operatorname{deg} D=2^{\ell} \quad \text { et } \quad \operatorname{deg} E=e \text {, }
$$

de sorte que $n=2^{\ell} e$. On a alors $A=D+E$ dans Br $k$ et vu l'hypothèse sur $E$,

$$
\frac{n}{2} \cdot A=\frac{n}{2} \cdot D \quad \text { dans } \operatorname{Br} k
$$

D'après les propriétés 3.1 , on a $T_{E}=e\langle 1\rangle$ dans $W k$ et $T_{A}=T_{D} \otimes T_{E}$, donc

$$
T_{A}=e T_{D} \quad \text { dans } W k .
$$

Si $\ell=0$, alors $\frac{n}{2} \cdot A=0$ dans $\mathrm{Br} k$ et $T_{A}=n\langle 1\rangle$ dans $W k$, donc la Proposition est claire. Si $\ell \geq 1$, on déduit de la relation (9) ci-dessus:

$$
C\left(T_{A}\right)=\epsilon \cdot C\left(T_{D}\right)+\frac{e(e-1)}{2} \cdot\left(\operatorname{disc} T_{D},-1\right)_{k} \quad \text { dans } \operatorname{Br} k .
$$

Or, la propriété $3.1(b)$ donne

$$
\operatorname{disc} T_{D}=\left\{\begin{array}{cc}
-1 & \text { si } \ell=1 \\
1 & \text { si } \ell>1
\end{array}\right.
$$

et vu le cas particulier déjà démontré on a.

$$
C\left(T_{D}\right)=2^{\ell-1} \cdot D+C\left(2^{\ell}\right) \quad \text { dans } \operatorname{Br} k
$$

Dès lors,

$$
C\left(T_{A}\right)=2^{\ell-1} \epsilon \cdot D+e \cdot C\left(2^{\ell}\right)+\left\{\begin{array}{cl}
\frac{e(e-1)}{2} \cdot(-1,-1)_{k} & \text { si } \ell=1 \\
0 & \text { si } \ell>1 .
\end{array}\right.
$$

Vu (8), la Proposition est démontrée, car un calcul direct montre que la somme des deux derniers termes est égale à $C(n)$.

\section{Références}

[1] J.K. Arason. Cohomologische Invarianten quadratischer Formen. J. Algebra 36 (1975) 448-491.

[2] T.Y. Lam. the Algebraic Theory of Quadratic Forms. Benjamin, Reading, Mass., 1973.

[3] D.W. Lewis. Trace forms of central simple algebras. Math. Z. 215 (1994) 367-375. 
[4] D.W. Lewis, J. Morales. The Hasse invariant of the trace form of a central simple algebra. Prépublication, 1993.

[5] A.S. Merkurjev, J.-P. Tignol. The multipliers of similitudes and the Brauer group of homogeneous varieties. Prépublication, 1993.

[6] C. Riehm. The corestriction of algebraic structures. Invent. Math. 11 (1970) 73-98.

[7] D.J. Saltman. Exposé à la Miniconference on Division Algebras and Quadratic Forms, Berkeley, October 1987.

[8] W. Scharlau. Quadratic and Hermitian Forms. Grundlehren math. Wiss. 270. Springer-Verlag, Berlin, 1985.

[9] J-P. Serre. Résumé des Cours et Travaux. Annuaire du Collège de France, 1990-1991.

[10] J-P. Tignol. Réduction de l'indice d'une algèbre simple centrale sur le corps des fonctions d'une quadrique. Bull. Soc. Math. Belg., Sér.A 42 (1990) 735-745.

Adresse usuelle:

Institut de Mathématique Pure et Appliquée Université Catholique de Louvain

chemin du cyclotron, 2

B-1348 Louvain-la-Neuve

Belgique 